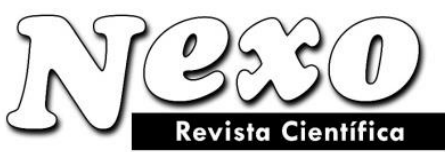

Vol. 34, No. 02, pp. 625-635/Junio 2021

\title{
Erosión hídrica superficial en la microcuenca del río Mancotal en Jinotega Nicaragua, 1996 - 2015
}

\author{
Surface water erosion in the Mancotal river micro-basin in Jinotega \\ Nicaragua, 1996 - 2015
}

\author{
Isolina C. Gutiérrez Aguilar*, Miguel E. Blanco Chávez \\ Universidad Nacional de Ingeniería. Programa de Investigación, Estudios Nacionales y Servicios del \\ Ambiente. Managua, Nicaragua. \\ *isolinagutierrez15@yahoo.com
}

(recibido/received: 03-noviembre-2020; aceptado/accepted: 22-febrero-2021)

\begin{abstract}
RESUMEN
La microcuenca del río Mancotal es un efluente del embalse Apanás que demanda información cuantitativa sobre la tasa de erosión de los suelos. La erosión de los suelos tiene un impacto negativo en la protección del embalse y se ha intensificado por el cambio del uso de vocación forestal a una agricultura de subsistencia. En este contexto, se estimó la erosión hídrica superficial mediante el uso de la Ecuación Universal Revisada de Pérdida de Suelo (RUSLE). Con este modelo se obtuvieron los factores de erosividad, erodabilidad, la longitud y el gradiente de la pendiente, cobertura del suelo y prácticas de conservación. También se encontró que existe una correlación aceptable $\left(\mathrm{r}^{2}=0.652\right)$, entre los valores de Erosividad de la lluvia $(R)$ y el Índice Modificado de Fournier $(I M F)$, por tanto, se puede estimar la erosividad utilizando los valores del IMF. Los resultados de la investigación mostraron que el $94.46 \%$ de la microcuenca presentó un potencial de erosión alto, superior a 25 Ton/ha/año.
\end{abstract}

Palabras claves: erodabilidad, longitud y gradiente de la pendiente, cobertura y prácticas de conservación.

\begin{abstract}
The Mancotal river micro-basin is an effluent from the Apanás reservoir that requires quantitative information about the rate of soil erosion. Soil erosion has a negative impact on the protection of the reservoir and has been intensified by the change in the use of forest-oriented land to subsistence farming. In this context, surface water erosion was estimated by using the "Revised Universal Soil Loss Equation (RUSLE)". With this model, the factors of erosivity, erodibility, the length and gradient of the slope, coverage of soil and conservation practices were obtained. It was also found that there is an acceptable correlation $\left(\mathrm{r}^{2}=0.652\right)$, between the rainfall erosivity and the Modified Fournier Index $(M F I)$ values, therefore, erosivity can be estimated using the $M F I$ values. The results of the investigation showed that $94.46 \%$ of the micro-basin presented a high potential erosion, greater than $25 \mathrm{Ton} / \mathrm{ha} /$ year.
\end{abstract}

Keywords: erodibility, length and gradient of the slope, coverage and conservation practices. 


\section{INTRODUCCIÓN}

El área de investigación es la microcuenca del río Mancotal, que por ser un efluente del embalse de Apanás, se enmarca en un creciente interés por obtener datos cuantitativos (Kothyari et al., 1994) de la erosión hídrica del suelo, información valiosa para apoyar la toma de decisiones para la protección del citado embalse.

Se ha observado que en el área de investigación, existe un cambio de uso de suelo de vocación forestal a una agricultura de subsistencia, que carece de prácticas de conservación de uso de suelo, en este sentido fue necesario generar información sobre erosión hídrica en la microcuenca, de tal manera que contribuya a dimensionar el problema, que permita tomar medidas oportunas de conservación del suelo para el funcionamiento óptimo del embalse y con ello contribuir al desarrollo sostenible de la energía hidroeléctrica del país.

En este contexto se utilizó el modelo matemático empírico Ecuación Universal Revisada de Pérdida de Suelo, RUSLE (Renard e. a., 1997), que ha sido ampliamente utilizado en muchas investigaciones en todos los continentes (Kumar, 2012), aun cuando los científicos han desarrollado numerosas herramientas para evaluar la pérdida de suelo.

Así con el modelo "RUSLE" y el sistema de información geográfico (ArcGIS), se estimó la tasa actual y potencial de la erosión hídrica superficial en la microcuenca del río Mancotal, subcuenca norte del lago de Apanás. Los resultados de estos productos se clasificaron según la FAO en tres niveles de amenaza de erosión de suelo.

\section{DISEÑO METODOLÓGICO Y DESARROLLO}

\subsection{Localización del área de estudio:}

El río Mancotal nace en el Cerro de Agua (1377 msnm) y desemboca en Apanás en la comarca Tomayunca $(957 \mathrm{msnm})$. La Figura 1, refleja las características de la microcuenca del río Mancotal, la cual se localizó entre los $13^{\circ} 12^{\prime} 57^{\prime \prime}$ a los $13^{\circ} 16^{\prime} 05^{\prime \prime}$ Latitud Norte y los $85^{\circ} 14^{\prime} 23^{\prime \prime}$ a los $86^{\circ} 02^{\prime} 37^{\prime \prime}$ Longitud Oeste, teniendo una extensión aproximada de $37.17 \mathrm{~km}^{2}$, abarcando parte de los territorios rurales del distrito VI de las comunidades Mancotal, San Gregorio y Tomayunca del municipio de Jinotega. Desde el punto de vista hidrológico, la microcuenca en estudio forma parte de la subcuenca norte del Lago Apanás que tiene una superficie de $588 \mathrm{~km}^{2}$, esta subcuenca es parte de la zona alta de la cuenca del río Grande de Matagalpa, que de acuerdo a la metodología PFAFSTETTER pertenece a la cuenca 9518.

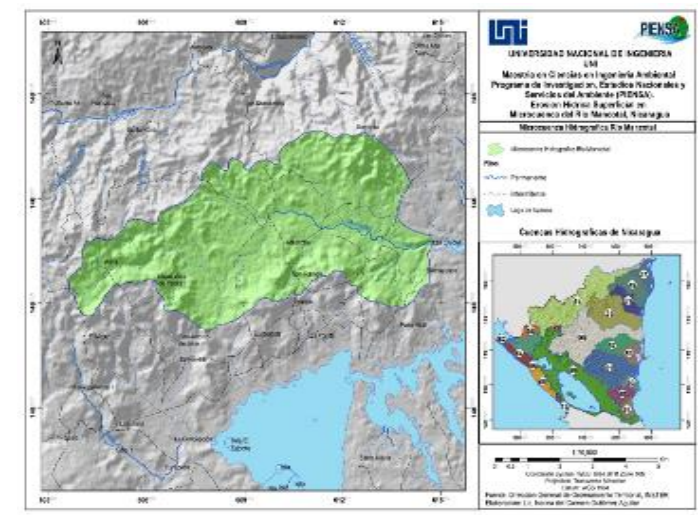

Figura 1. Microcuenca Mancotal, con un área de 37.17 km². 


\subsection{Análisis de los datos de precipitación.}

La característica de las 10 estaciones meteorológicas seleccionadas se muestra en la Tabla 1 (INETER, 1998). Estas estaciones presentaron más del $80 \%$ de sus registros completos, en el período de estudio de 1996 al 2015. Antes de utilizar esta información fue rellenada por correlación y regresión lineal múltiple y comprobada su consistencia por el método de doble masa, además se determinó que las observaciones de la variable climatológica precipitación, fueron medidas con los mismos instrumentos meteorológicos (pluviómetro y pluviógrafos), sin cambios en su emplazamiento local, ni en el procedimiento de observación, por lo cual los datos recopilados son homogéneos, es decir válidos y confiables en el período que se tomó como referencia.

Tabla 1. Estaciones representativas del área de investigación.

\begin{tabular}{|c|c|c|c|c|c|c|}
\hline Código & Estación & Municipio & Latitud (N) & $\begin{array}{l}\text { Longitud } \\
\text { (W) }\end{array}$ & $\begin{array}{l}\text { Altitud } \\
\text { (msnm) }\end{array}$ & Tipo \\
\hline 45015 & $\begin{array}{l}\text { San } \\
\text { Sebastián de } \\
\text { Yalí }\end{array}$ & $\begin{array}{l}\text { San } \\
\text { Sebastián de } \\
\text { Yalí }\end{array}$ & $13^{\circ} 18^{\prime} 18^{\prime \prime}$ & $86^{\circ} 11^{\prime} 00^{\prime \prime}$ & 880 & PV \\
\hline 45018 & Las Praderas & $\begin{array}{l}\text { Santa María } \\
\text { de Pantasma }\end{array}$ & $13^{\circ} 20^{\prime} 54^{\prime \prime}$ & $85^{\circ} 56^{\prime} 48^{\prime \prime}$ & 415 & PV \\
\hline 55001 & $\begin{array}{l}\text { Corinto } \\
\text { Finca }\end{array}$ & Jinotega & $13^{\circ} 10^{\prime} 11^{\prime \prime}$ & $85^{\circ} 53^{\prime} 00^{\prime \prime}$ & 1070 & PV \\
\hline 55006 & Los Robles & Jinotega & $13^{\circ} 10^{\prime} 00^{\prime \prime}$ & $85^{\circ} 57^{\prime} 06^{\prime \prime}$ & 990 & $\mathrm{HMO}$ \\
\hline 55019 & Mancotal & Jinotega & $13^{\circ} 14^{\prime} 36^{\prime \prime}$ & $85^{\circ} 54^{\prime} 06^{\prime \prime}$ & 950 & HMO \\
\hline 55020 & Jinotega & Jinotega & $13^{\circ} 05^{\prime} 06^{\prime \prime}$ & $85^{\circ} 59^{\prime} 48^{\prime \prime}$ & 1032 & HMP \\
\hline 55041 & $\begin{array}{l}\text { Los } \\
\text { Horcones }\end{array}$ & $\begin{array}{l}\text { San Rafael } \\
\text { del Norte }\end{array}$ & $13^{\circ} 13^{\prime} 00^{\prime \prime}$ & $86^{\circ} 04^{\prime} 00^{\prime \prime}$ & 1320 & PV \\
\hline 55043 & La Porfía & Jinotega & $13^{\circ} 08^{\prime} 48^{\prime \prime}$ & $86^{\circ} 03^{\prime} 12^{\prime \prime}$ & 970 & $\mathrm{HMO}$ \\
\hline 55044 & La Porra & Jinotega & $13^{\circ} 15^{\prime} 12^{\prime \prime}$ & $85^{\circ} 59^{\prime} 12^{\prime \prime}$ & 1020 & PV \\
\hline 69021 & $\begin{array}{l}\text { San Rafael } \\
\text { del Norte }\end{array}$ & $\begin{array}{l}\text { San Rafael } \\
\text { del Norte }\end{array}$ & $13^{\circ} 13^{\prime} 00^{\prime \prime}$ & $86^{\circ} 07^{\prime} 00^{\prime \prime}$ & 1078 & PV \\
\hline
\end{tabular}

\subsection{Método RUSLE.}

Los suelos a nivel de orden se clasificaron, según (INETER, 2015) y la erosión hídrica superficial se estimó con (1). Los diferentes mapas utilizados y los resultantes están a escala 1:70000, donde la interpolación de datos se realizó con inverso de la distancia en ArcGis 10.1.

$$
A=R K L S C P
$$

Donde:

$A=$ Es la pérdida de suelos en Ton/(ha.año)

$R=$ El factor erosividad de la lluvia, en MJ.mm/(ha.hr)

$K=$ El factor erodabilidad del suelo en Ton/(ha.año)/(MJ.mm/(ha.hr))

$L=$ El factor de longitud de la pendiente (adimensional).

$S=$ El factor pendiente (adimensional). 
$C=\mathrm{El}$ factor cobertura y manejo de la vegetación (adimensional).

$P=$ El factor de prácticas de conservación (adimensional).

Estimación del Factor erosividad de la lluvia (R): Dado que en la zona de estudio se disponía de sólo una estación pluviográfica con registros de precipitación cada 5 minutos (estación Jinotega), se determinó los valores de $R$ anual y los valores del Índice Modificado de Fournier (IMF) para esa estación. Luego se correlacionaron los valores anuales de $R$, con el $I M F$ en la estación pluviográfica de Jinotega, obteniéndose (2), con un nivel de significancia del 5\%. Los resultados de la correlación, muestran un coeficiente de determinación significativo $\left(\mathrm{r}^{2}=0.652\right)$, por lo cual (2) es aceptable. Algunos autores como (Renard \& Freidmund, 94), (Rivera \& Gomez, 1991), (Saldaña Diaz, Nemmaoui, Cantón Castilla, Aguilar Torrez, \& Aguilar Torrez, 2014) y (Blanco, 2019) han desarrollado relaciones similares para estimar R a partir de datos de precipitación. Posteriormente se utilizó (2) para estimar los valores de $R$ anual en las nueve estaciones pluviométricas del área de investigación. La severidad de los valores mencionados fue clasificada según los criterios de (Rivera, P.J.H. y A.A. Gómez, 1991).La del IMF y la erosividad de la lluvia anual (R) se muestra en la Figuras 2(a) y 2(b).

$$
\begin{gathered}
R=505.47+12.229(I M F) \\
\mathrm{R}=\sum \mathrm{EI}_{30}
\end{gathered}
$$

Donde,

$R=$ Índice de erosividad de la lluvia (MJ/ha- $\mathrm{mm} / \mathrm{h})$

$\mathrm{E}=$ Energía Cinética total de la tormenta $(\mathrm{MJ} / \mathrm{ha})$

$\mathrm{I}_{30}=$ Intensidad máxima de la tormenta en 30 minutos $(\mathrm{mm} / \mathrm{h})$

$$
I M F=\sum_{i=1}^{i=12} \frac{p_{i}^{2}}{p_{t}}
$$

Donde,

$I M F=$ índice modificado de Fournier

$i=$ número del mes.

$P i=$ precipitación mensual en $\mathrm{mm}$.

$P t=$ precipitación promedio anual en $\mathrm{mm}$.

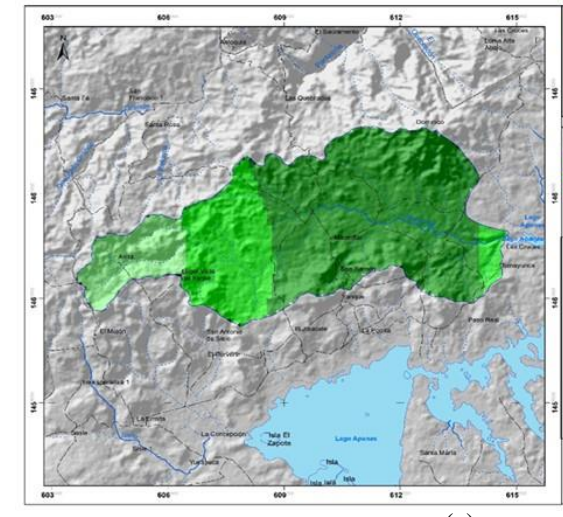

(a)
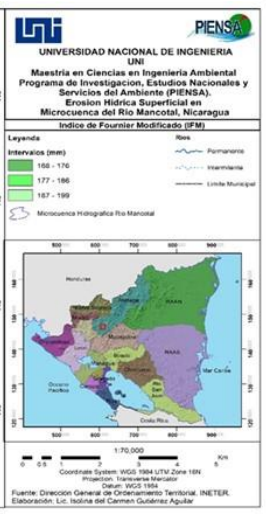

on

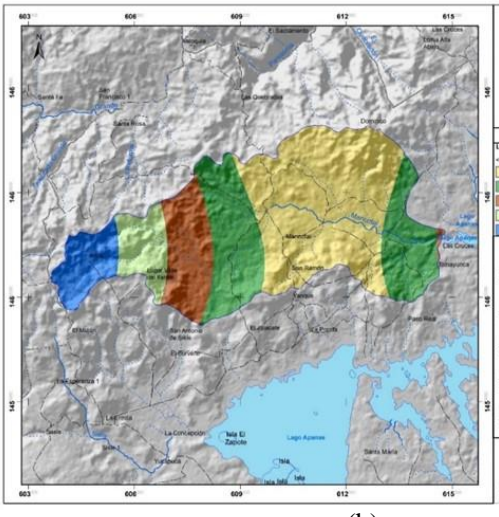

(b)

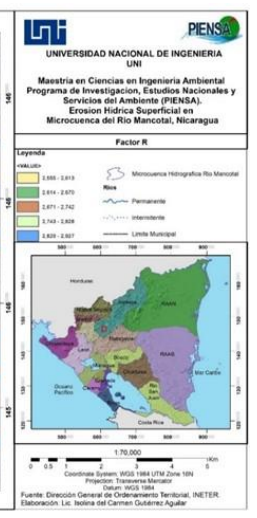

Figura 2.(a) Índice Modificado de Fournier IMF. (b) Factor erosividad de la lluvia R 
Factor erodabilidad del suelo $(K)$ : El factor de erodabilidad del suelo $(\mathrm{K})$ se define como la tasa de pérdida de suelo por unidad de energía erosiva creada por la lluvia y calculada en condiciones estándar para una parcela experimental ( $22.1 \mathrm{~m}$ largo x $3.6 \mathrm{~m}$ ancho) que consiste en suelo limpio y desnudo con una pendiente del 9\% (Renard e. a., 1997). Este factor indica el grado de sensibilidad o resilencia del suelo a la erosión y se concibe como la facilidad con la cual es desprendido por el salpicado de la gota durante un evento lluvia, el flujo superficial o bien por la acción de ambos fenómenos (Montes M. A., 2011).

Enmarcado en este proceso se tomaron muestras de suelo en campo y fueron analizadas en el laboratorio de la Universidad Nacional Agraria, del cual se obtuvo por el método de Pipeta de Robinson la textura, con el análisis de WalkleyBlack los porcentajes de materia orgánica, mediante observación sensorial (visual y tacto) la estructura y con el código la categoría de permeabilidad del suelo. Con esta información (Tablas 2 y 3 ) se estimó con (5) el factor $K$.

$$
100 K=2.1 *\left(M^{1.14} / 10,000\right) *(12-O M)+3.25(s-2)+2.5(p-3)
$$

Donde:

$M=$ Limo+ arena muy fina $(\%)(100-\%$ arcilla $)$

$O M=$ materia orgánica

$\underline{\mathrm{s}}=$ clase de estructura

$p=$ clase de permeabilidad

Tabla 2. Resultado de laboratorio de las muestras de suelo de la microcuenca Mancotal.

\begin{tabular}{ccccccccccc}
\hline Cod & $\begin{array}{c}\text { Orden } \\
\text { de } \\
\text { Suelo }\end{array}$ & Estructura & $\begin{array}{c}\text { Permeabilida } \\
\text { d }\end{array}$ & $\begin{array}{c}\text { \%Arena } \\
\text { muy fina } \\
(63 \mathrm{Nm})\end{array}$ & $\begin{array}{c}\text { Aren } \\
\text { a } \\
\text { Total }\end{array}$ & $\begin{array}{c}\text { Limo } \\
\text { Grueso }\end{array}$ & $\begin{array}{c}\text { Limo } \\
\text { Fino }\end{array}$ & $\begin{array}{c}\text { Limo } \\
\text { Total }\end{array}$ & $\begin{array}{c}\% \\
\text { Arcilla }\end{array}$ & $\begin{array}{c}\text { Clase } \\
\text { Textura }\end{array}$ \\
\hline 826 & Alfisol & $\begin{array}{c}\text { Granular } \\
\text { compuesto } \\
\text { Granular } \\
\text { compuesto }\end{array}$ & $\begin{array}{c}\text { Moderamente } \\
\text { lenta }\end{array}$ & 0.29 & 10.75 & 3.44 & 25.8 & 29.24 & 60.01 & Arcilloso \\
Moderada & 0.611 & 22.47 & 7.04 & 27.34 & 34.38 & 43.15 & Arcilloso \\
\hline
\end{tabular}

Tabla 3. Valores de los cinco parámetros para determinar el Factor $K$.

\begin{tabular}{|c|c|c|c|c|c|c|c|c|c|}
\hline \multirow[b]{2}{*}{ Cod } & \multirow[b]{2}{*}{$\begin{array}{l}\text { Tipo de } \\
\text { Suelo }\end{array}$} & \multirow[b]{2}{*}{$\begin{array}{l}\text { Clase } \\
\text { Textura }\end{array}$} & \multicolumn{2}{|c|}{ Código } & \multirow{2}{*}{$\begin{array}{l}\text { Químico } \\
\text { MO (\%) }\end{array}$} & \multicolumn{4}{|c|}{$\begin{array}{l}\text { Granulometría } \\
\text { Partículas }\end{array}$} \\
\hline & & & Estructura & Permeabilidad & & $\begin{array}{l}\% \text { Arena } \\
\text { muy fina } \\
(63 \mathrm{Nm})\end{array}$ & $\begin{array}{c}\% \\
\text { Limo } \\
\text { Total }\end{array}$ & $\begin{array}{c}\% \\
\text { Arcilla }\end{array}$ & $\begin{array}{c}K \\
\text { T/ha-año/MJ-mm/ha-h }\end{array}$ \\
\hline 826 & Alfinsols & Arcilloso & 3 & 4 & 5.62 & 0.29 & 29.24 & 60.01 & 0.010 \\
\hline 827 & Ultisols & Arcilloso & 3 & 3 & 4.97 & 0.611 & 34.38 & 43.15 & 0.020 \\
\hline
\end{tabular}

Con esta información y los datos de nivel de orden uso de los suelos se realizó el mapa de erodabilidad $(K)$ del suelo (Figura 3).

Longitud de pendiente y factor de inclinación $(L S)$ : La erosión del suelo está influenciada por la topografía, fundamentalmente por la longitud $\boldsymbol{L}$ y la inclinación de la pendiente $\boldsymbol{S}$. El factor $\boldsymbol{L}$ estimado con (6) (Wischmeier \& Smith, 1978), fue modificada por (PJJ Desmet, 1996) para un manejo SIG considerando la acumulación de flujo y agregando una relación de erosión entre surcos y surcos (7), mientras que el factor $\boldsymbol{S}$, se estimó con método de (DK McCool, 1987) expresado en (8) - (11). Ambos 
algoritmos están integrados en la herramienta de análisis espacial ArcGIS 10.1, en el Modelo de Elevación Digital de AlosPalsar DEM. Con esta información se realizó el mapa ráster de factor $\boldsymbol{L} \boldsymbol{S}$ (Figura 4).
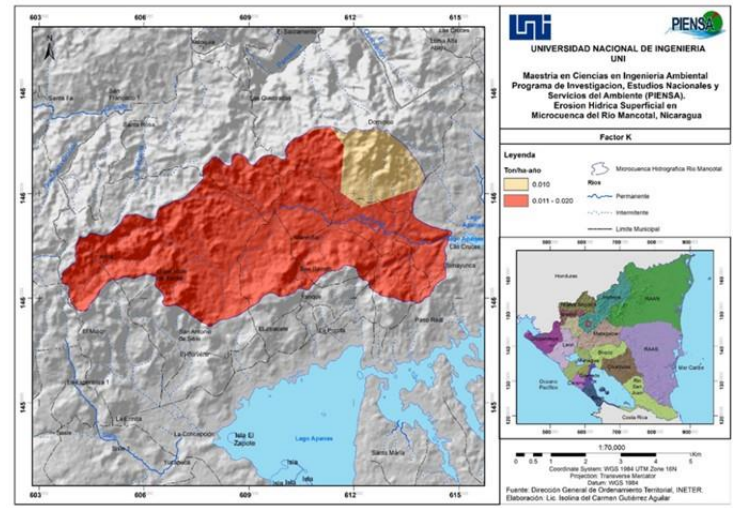

Figura 3. Mapa del factor de erodabilidad (K) del suelo en la microcuenca de Mancotal.

$$
\begin{gathered}
L=(\lambda / 22.13)^{m} \\
L_{i, j}=\frac{\left(A_{i, j-i n}+D^{2}\right)^{m+1}-A_{i . j-i n}^{m+1}}{D^{m+2} * X_{i, j}^{m} * 22.13^{m}}
\end{gathered}
$$

Donde $A(\mathrm{i}, \mathrm{j})[\mathrm{m}]$ es el área aportadora unitaria a la entrada de un pixel (celda), $D$ es el tamaño del pixel y $X$ es el factor de corrección de forma.

$$
m=\frac{\beta}{(1+\beta)}
$$

Donde $\boldsymbol{m}$ representa la relación entre surcos y surcos se calcula con la siguiente expresión:

$$
\begin{gathered}
\beta=\left\{\left((\operatorname{sen} \theta) /\left(3,0 *(\operatorname{sen} \theta)^{0.8}\right)+0.56\right)\right\} * r \\
S=16.8 \operatorname{sen} \theta-0,50 \text { si la pendiente } \geq 9 \% \\
S=10.8 \operatorname{sen} \theta-0,03 \text { si la pendiente }<9 \%
\end{gathered}
$$

Donde

$S$ : Factor de inclinación de la pendiente.

$\theta$ : Ángulo de inclinación en grado.

$r$ : Coeficiente igual a:0.5 en tierras forestales o pastizales; 1.0 en terrenos agrícolas y 2.0 en sitios en construcción.

Factor cobertura vegetal $(C)$ : Para obtener la cobertura en la microcuenca con ayuda del sistema de información geográfica, se identificó el tipo de vegetación y utilizando los valores de cobertura de (Rosse, 1977). Con base en esta información se elaboró el mapa ráster del factor $C$ (Figura 5). 


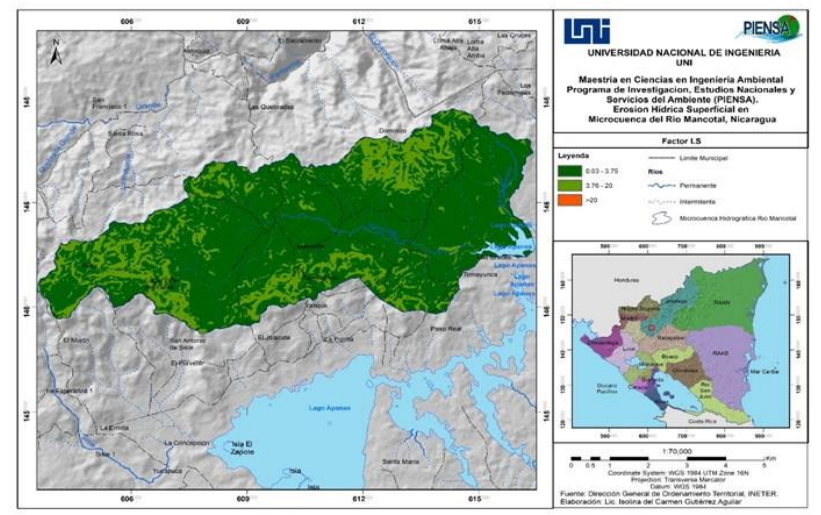

Figura 4. Mapa del factor $L S$, longitud y pendiente del suelo en la microcuenca de Mancotal.

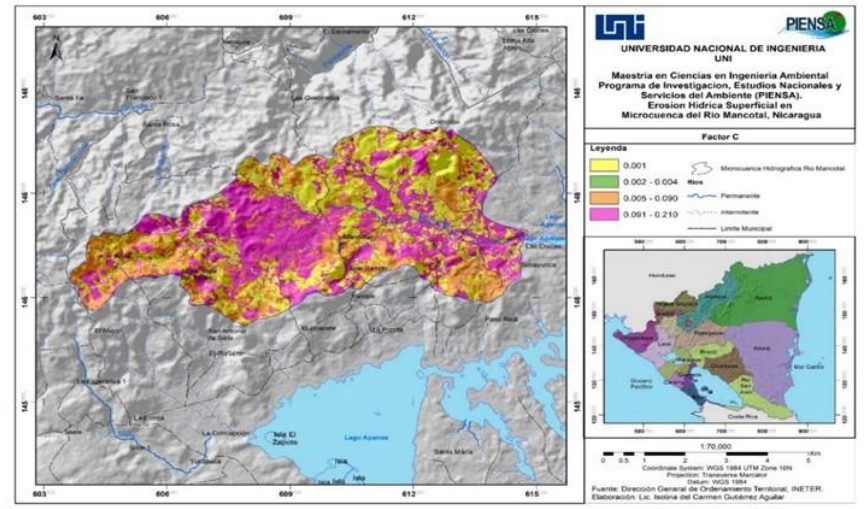

Figura 5.Factor $C$, cobertura y manejo de la vegetación en la microcuenca del río Mancotal.

Factor prácticas de conservación de suelos $(P)$ : El Factor de prácticas de conservación de suelo es la relación entre pérdida de suelo con una práctica de conservación específica y la pérdida correspondiente con labranza en pendiente ascendente y descendente, en áreas principalmente agrícola tales como cultivos en terraza, curvas de nivel, etc.

Actualmente no se dispone a escala nacional, con la cartografía de áreas agrícolas, con prácticas de manejo de conservación, por lo cual los valores del factor $P$ se obtuvieron del mapa uso de suelo y de los valores de factor $P$ para cultivos en faja, según (Wischmeier \& Smith, 1978). Asignándole a las áreas ocupadas por bosque, pasto y arboles dispersos, el valor de $P$ igual a 1 y por la agricultura $P$ igual a 0.30 . Con base en esta información se elaboró el mapa ráster del factor $\mathrm{P}$ (Figura 6).

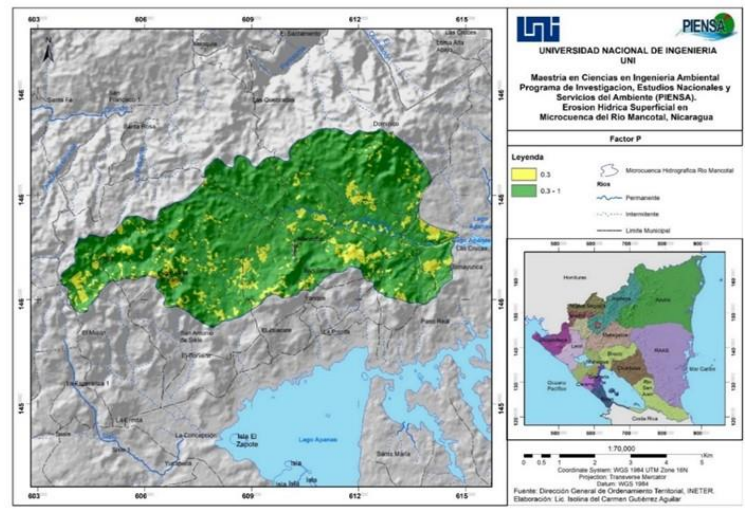

Figura 6. Mapa del factor $P$ : Prácticas de conservación del suelo en la microcuenca de Mancotal. 


\section{RESULTADOS}

Los valores de la erosión hídrica actual y la erosión hídrica potencial, se estimaron con (1), pero en el cálculo de la erosión potencial se consideraron solamente factores naturales (RKLS). Los valores obtenidos se clasificaron según la FAO (1985) en tres niveles de amenaza (baja, media y alta) de erosión de suelo. Toda esta información se trabajó en ambiente SIG (Arc View 10.1), donde se sobrepusieron las diferentes capas de erosividad, erodabilidad, pendiente, cobertura y manejo para obtener los mapas de erosión hídrica actual y potencial (Figura 7) de la microcuenca Mancotal.

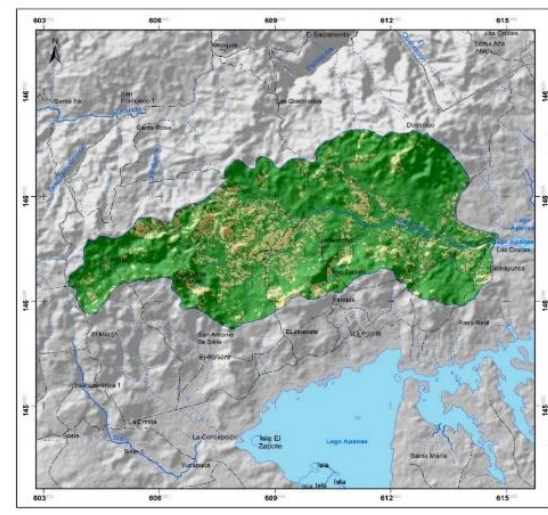

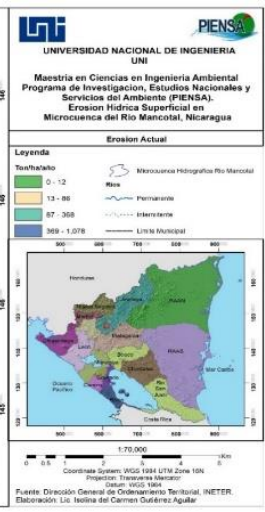

(a)

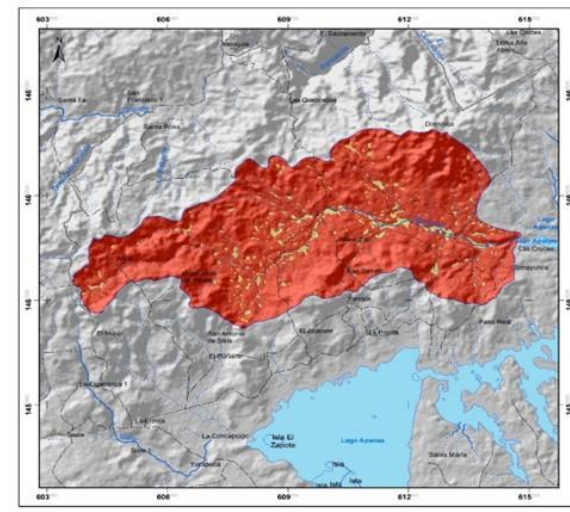

(b)

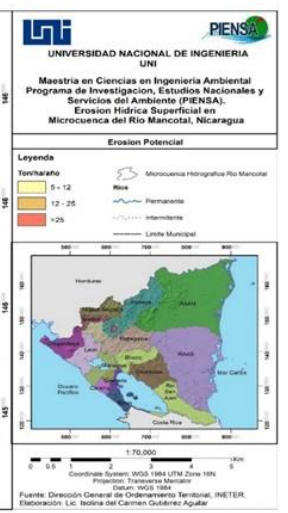

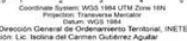

Figura 7.(a) Erosión Actual del suelo. (b) Erosión Potencial del suelo

\subsection{Erosión actual y potencial del suelo}

Del área total de la microcuenca Mancotal, se tiene que el 78.4\% de la superficie, predomina una erosión actual baja de 12 t/ha/año, mientras que en el $21.6 \%$ del área muestra una erosión actual alta de 13 a 86 t/ha/año (Figura 7a). Esto podría ser un problema de alto riesgo, debido a que la erosión media anual, según el mapa de uso actual, está cambiando con la pérdida de suelo de vocación forestal por la agricultura de subsistencia, lo que aumenta los riesgos de erosión y arrastre de sedimento al embalse de lago de Apanás.

La estimación de la erosión potencial se realizó con los factores naturales de (1), es decir sin considerar los componentes antrópicos, los valores obtenidos se clasificaron en tres niveles de riesgo de erosión potencial, de acuerdo con los limites determinados por (INETER - COSUDE, 2005). Esta clasificación se efectuó con la finalidad de representar el efecto que ha tenido la erosión hídrica en el área y para identificar rápidamente las zonas más afectadas.

Enmarcados en la clasificación antes mencionada, se tiene que, del total del área de investigación, $94.46 \%$, presenta una erosividad potencial alta, con valores superiores a $25 \mathrm{t} / \mathrm{ha} / \mathrm{año}$, el $0.06 \%$, corresponde a la erosión potencial media con valores que oscilaron de 12 a 25 t/ha/año y el $5.48 \%$ muestra una erosión potencial baja entre 5 a 12 t/ha/año (Figura 7b).

\section{CONCLUSIÓNES}

En la investigación realizada se encontró que el 78.4\% de la microcuenca Mancotal, presentó una erosión actual baja de 12 t/ha/año y un $21.6 \%$ con erosión alta de 13 a 86 t/ha/año. También se estimó que el $94.46 \%$ de la superficie de la microcuenca, mostró una erosión potencial alta superior a 25 t/ha/año. Ambos resultados exceden a la tolerancia de erosión de suelo propuesta por Wischmeier y Smith, a causa 
del cambio de uso de suelo con vocación forestal, pues se encontró que el $11.8 \%$ del área de la cuenca está sufriendo cambios bruscos por una agricultura de subsistencia.

También se encontró que el uso de técnicas de conservación de suelo es nulo, conduciendo a que la preparación del suelo en pendientes mayores de $14 \%$ pueden incrementar bruscamente los riesgos de erosión superficial, afectado el desprendimiento y transporte de sedimento al embalse de Apanás.

Además, se determinó que el valor de erosividad de la lluvia $(R)$ y los valores del Índice Modificado de Fournier $(I M F)$ tienen buena correlación lineal, con un coeficiente de determinación $\mathrm{r}^{2}=0.652$, lo que demuestra que se puede estimar la erosividad $R$ utilizando el $I M F$.

\section{REFERENCIAS}

Baca, G. (2007). Fundamentos de Ingeniería Económica (4a. ed.). México D.F.: McGraw-Hill.

Blanco, M. E. (2019). Factor Erosividad de la lluvia en la subcuecna Sur del lago Xolotlan, Managua. Nexo Revista Cientifica, 32(1), 51-51.

DK McCool, L. B. (1987). Factor de pendiente revisado para la ecuación universal de pérdida de suelo. Trans, ASAE, 30, 1387-1396.

Garza, J. (2014). Análisis multicriterio de puntos de inflexión de precio en el mercado de divisas. San Nicolás de los Garza, Nuevo León: Universidad Autónoma de Nuevo León.

Gomero, N. (2014). Análisis económico de los impuestos: Impacto en la rentabilidad de las inversiones. Quipukamayoc, 79-87.

INETER - COSUDE. (2005). Recomendaciones Técnicas para la Elaboración de Mapa de Amenaza por Erosión Hídrica. Managua, Nicaragua: Autor.

INETER. (1998). Catálogo de Estaciones Meteorológica de Nicaragua. Managua, Nicaragua: Autor.

INETER. (2015). Mapa de Suelos de la República de Nicaragua. Managua, Nicaragua: Autor. Instituto Nicaragüense de Investigaciones y Estudios Tributarios. (2015). Balance de la Ley de Concertación Tributaria. Managua, Nicaragua: INIET.

Kheir et al. (2008). Evaluación de la erosión del suelo en los paisajes kársticos mediterráneos del Líbano utilizando la teledetección y los SIG. Engineering Geology, 99, págs. 239 - 254.

Kothyari et al. (1994). Predicción del rendimiento de sedimento. Ingeniería de Riego y Drenaje, pp. 1122 - 1131 mil.

Kumar, P. R. (2012). Enfoque integrado de la ecuación universal de pérdida de suelo (USLE) y el sistema de información geográfica (SIG) para la evaluación del riesgo de pérdida de suelo en el sur del sur de la Cuenca Koel, Jharkhand. Revista del Sistema de Información Geográfica , 4 ( 6 ), pp. 588 - 596. 
La Gaceta. Diario Oficial. (2013). Decreto 01-2013. Reglamento de la Ley No. 822, Ley de Concertación Tributaria. Managua: La Gaceta.

Montes M. A., E. M.-C. (2011). Mapa de Erosión Potencia. Tecnología y Ciencias del Agua, vol II., 5-17.

PJJ Desmet, G. G. (1996). Un procedimiento SIG para calcular automáticamente el factor USLE LS de paisaje topográfico comlejo. J. Conserv de agua del suelo. ,51, 427- 433.

Renard, e. a. (1997). Predicción de la erosión del suelo por el agua: una guía para la planificación de la conservación con la ecuación universal revisada de pérdida de suelo (RUSLE).Manual de Agricultura $N^{\circ} 703$. Washington D.C: (USDA).

Renard, K. G., \& Freidmund, J. R. (94). Using monthly precipitation data to estimate the R factor in the revised USLE. Journal of Hydrology, 15(7), 287-306.

Rivera, J. H., \& Gomez, A. A. (1991). Erosividad de la lluvia en la zona cafetalera central Colombiana (Caldas, Quindo y Risaralda). Cenicafe, 42(2), 37-52.

Rivera, P.J.H. y A.A. Gómez. (1991). Erosividad de las lluvias en las zonas cafeteras central Colombiana (Caldas, Quindio y Risaralda). CENICAFE,42 (2), pp. 37-52.

Rosillo, J. (2005). La inflación: ¿Elemento inocuo en las decisiones de inversión? Forum Empresarial, 20-46.

Rosse, E. e. (1977). Uso de la ecuación universal de pérdida de suelo para predecir la erosión en África occidental", en Erosión del suelo: predicción y control, Actas de la Conferencia Nacional sobre Erosión del Suelo, Soil Conservation Society of America. Iowa: Ankeney pp. 143-151.

Ruiz, J. (1992). Capital y depreciación: Una aproximación endógena. Valencia, España: Universitat de Valencia.

Saldaña Diaz, M., Nemmaoui, A., Cantón Castilla, M. Y., Aguilar Torrez, M. A., \& Aguilar Torrez, F. J. (2014). Estimacion de la erosion potencial en la cuena del rio Moulouya aguas arriba de la presa Mahamend V. MAPPING, 23, 4-16.

Wischmeier \& Smith. (1978). Predicción de pérdidas por erosión por lluvia: Una guía para la planificación de la conservación.Manual de Agricultura No. 537 USDA. Washington DC, Estados Unidos de América: Autor.

\section{SEMBLANZA DE LOS AUTORES}

Isolina C. Gutiérrez Aguilar: Obtuvo el grado de Licenciatura en Ciencias de la Educación con mención en Matemática en la Universidad Autónoma de Nicaragua. Desarrolló sus estudios de Maestría en Ciencias Ambiental en el Programa de Investigación y Docencia en Medio Ambiente (PIDMA) de la UNI, Nicaragua. Trabajo actual como Coordinador de Área Técnica en la Dirección General de Meteorología del Instituto Nicaragüense de Estudios Territoriales INETER. 
Miguel E. Blanco Chávez: Obtuvo el grado de Ingeniero Civil en la Universidad Nacional de Ingeniería, Nicaragua. Desarrolló sus estudios de Maestría en Ingeniería Ambiental en el Programa de Investigación y Docencia en Medio Ambiente (PIDMA) de la UNI, Nicaragua. Ha participado como consultor en proyectos hidroeléctricos, de amenazas por inundación, de drenaje pluvial y manejo de cuencas hidrográficas. Ha sido profesor de hidráulica e hidrología en la carrera de ingeniería civil en la FTC de la UNI. Actualmente imparte los cursos Hidrología Aplicada y Redes de Alcantarillado en el Programa de Investigación, Estudios Nacionales y Servicios del Ambiente (PIENSA) de la UNI, Nicaragua. 